\title{
Notas sobre la familia Gelastocoridae (Hemiptera: Heteroptera) en el extremo sur de Chile, con descripción de un subgénero y especie nuevos
}

\author{
Notes on the family Gelastocoridae (Hemiptera: Heteroptera) in \\ Southern Chile with descriptions of a new subgenus and a new species
}

Eduardo I. Faúndez ${ }^{1,3}$ \& Allan C. Ashworth²

\section{Resumen}

Se describe Nerthra subantarctica Faúndez \& Ashworth sp. nov., a partir de material fósil de Puerto Edén ( 49 $\left.{ }^{\circ} \mathrm{S}\right)$, Provincia de Última Esperanza, Región de Magallanes, Chile, en base a una cabeza y pronoto. A pesar de ser una especie descrita en base a material fósil, se cree que esta especie no se encuentra extinta, dada su poca antigüedad. Se entregan nuevos registros y se da a conocer el primer macho para Nerthra praecipua Todd, 1957. Se erige el nuevo subgénero Rhinodermacoris Faúndez subgen. nov., para ubicar a $N$. praecipua. La distribución de esta especie se amplía hacia el norte alcanzando la Región de los Ríos ( 40 ${ }^{\circ} \mathrm{S}$ ); mientras que $N$. subantarctica representaría el registro más meridional conocido para Gelastocoridae en el continente Americano.

\section{Palabras clave:}

Nerthra, Rhinodermacoris, nueva especie, nuevo subgénero, Magallanes.

\footnotetext{
Abstract

A fossil gelastocorid, Nerthra subantarctica Faúndez \& Ashworth sp. nov, is described from Puerto Édén ( 49 ${ }^{\circ}$ S), Última Esperanza province, Magallanes Region, Chile. The new species is described on basis of a head and a pronotum. Although it is a fossil species it is not believed to be extinct. New records and first description of the male are given for Nerthra praecipua Todd, 1957, and a new subgenus Rhinodermacoris Faúndez subgen. nov., is erected to place $N$. praecipua. The
}

male of $N$. praecipua is described for the first time. The new records for $N$. praecipua extends its north distribution up to Los Ríos Region ( 40 $\left.{ }^{\circ} \mathrm{S}\right)$. On the other hand $N$. subantarctica is the southernmost distributed gelastocorid in South America.

\section{Key words:}

Nerthra, Rhinodermacoris, new species, new subgenus, Magallanes.

\section{INTRODUCCIÓN}

Los gelastocóridos son una familia de heterópteros que comprende 3 géneros y 111 especies a nivel mundial (Henry, 2009); no obstante análisis recientes indican que la cantidad de géneros presentes dentro de esta familia debería aumentar (Cassis, 2006).

Estos insectos son comúnmente conocidos como "chinches sapo" o "sapitos de barro", debido a su parecido con especies de la familia Bufonidae (Amphibia), a su capacidad de saltar, y a que suelen encontrarse en ambientes ribereños.

Este grupo fue revisado por Todd (1955),

1 Entomology Department, School of Natural Resource Sciences, North Dakota State University, Dept. 7650, P.O. Box 6050; Fargo, ND, USA. ed.faundez@gmail.com $\longrightarrow$

2 Geosciences Department, North Dakota State University, Dept. 6050, P.O. Box 6050; Fargo, ND, USA.

3 Departamento de Zoología Médica, Centro de Estudios en Biodiversidad (CEBCh), Magallanes 1979, Osorno, Chile. 
y actualmente está representado en Chile por 9 especies, 3 de ellas en el género Gelastocoris y las restantes 6 pertenecen al género Nerthra. Mayoritariamente los gelastocóridos chilenos se distribuyen en la zona centro-sur del país.

La presente contribución tiene por objeto describir una nueva especie y un nuevo subgénero de esta familia, entregando una actualización taxonómica y distribucional para el extremo sur de Chile.

\section{MATERIALES Y MÉTODOS}

Las fotografías fueron tomadas con una cámara digital adaptada a un microscopio estereoscópico. En morfología se sigue a Todd (1955). Las colecciones mencionadas en el texto son las siguientes: IDEP (Pabellón de Colecciones Biológicas "Prof. Edmundo Pisano", Instituto de la Patagonia, Universidad de Magallanes Chile), MUEC (Museo Entomológico Luis Peña, Departamento de Sanidad Vegetal, Universidad de Chile), EIFC (Eduardo I. Faúndez). La edad del fósil fue estimada con una secuencia de dataciones de radiocarbono, a partir del perfil de la turba (Ashworth et al. 1991). Todas las medidas se encuentran en milímetros.

\section{RESULTADOS Y DISCUSIÓN}

Nerthra subantarctica Faúndez \& Ashworth sp. nov. (Figs. 1, 2)

\section{Descripción}

Cabeza (Fig. 1): Anaranjado-parduzco, triangular en vista frontal, textura verrugosa $y$ superficie irregular, ápice redondeado; ojos muy prominentes parduzcos, subovalados; ocelos muy pequeños, rojo parduzco, cada uno situado sobre una tumescencia; sin tubérculos superapicales o laterales.

Pronoto (Fig. 2): Anaranjado-parduzco, subrectangular, textura verrugosa; disco elevado, compuesto de dos lóbulos claramente marcados en su borde apical, borde anterior levemente convexo; lados ligeramente convexos y curvados hacia el interior en los ángulos anterolaterales; ángulos anterolaterales truncados; ángulos humerales ligeramente redondeados, sin proyectarse lateralmente; base del pronto con un lóbulo convexo y redondeado a cada lado de la línea media, centro levemente cóncavo.

\section{Medidas}

Largo de la cabeza: 1,42; ancho de la cabeza: 3,05; diámetro ocular: 0,72; distancia interocellar: 0,76; largo del pronoto: 1,97; ancho del pronoto: 4,11 .

\section{Holotipo}

Chile, Región de Magallanes, Isla Wellington, Puerto Edén, en pequeño pantano al oeste de Villa Puerto Edén, 49,131546 'S, $74,432524{ }^{\circ} \mathrm{O}$, A. Ashworth leg. Cabeza y pronoto montados en placa, números 6 y 7 , extraídos en un perfil de turba a unos $15-20 \mathrm{~cm}$ de profundidad desde la superficie del pantano [IDEP]. Fósil con una datación de 2.800 edades de radiocarbono, las que equivalen a aproximadamente 2.865 años A. P.
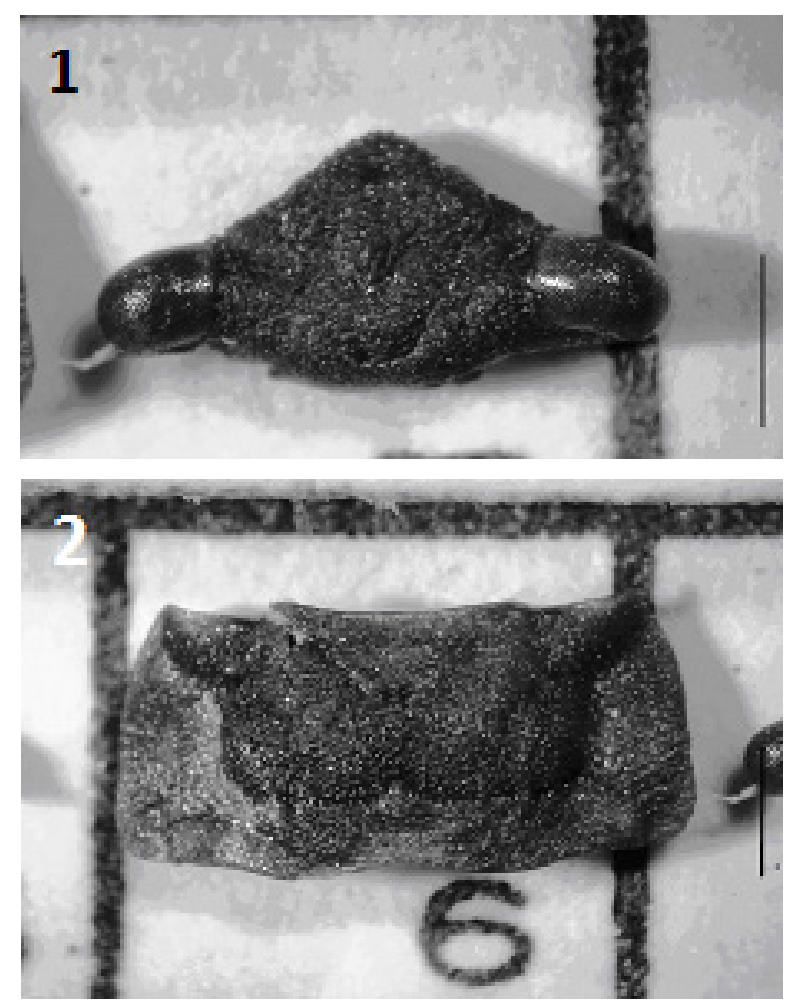

Figuras 1 y 2. Nerthra subantarctica Faúndez \& Ashworth, sp. nov. escala $=1 \mathrm{~mm}, 1$. Cabeza; 2. Pronoto 


\section{Etimología}

Nombre específico: subantarctica, debido a que es una especie endémica de la subregión subantarctica (Skottsberg, 1905).

\section{Comentario}

Esta especie es cercana a Nerthra parvula (Signoret, 1864) y Nerthra undosa Nieser \& Chen, 1992, de las que se diferencia por la forma del pronoto (más delgado y rectangular, con los ángulos anterolaterales no proyectados anteriormente en $N$. subantarctica), y la cabeza que no presenta tubérculos; adicionalmente los ocelos son notoriamente más reducidos que en las otras especies presentes en Chile. Pese a ser una especie descrita a partir de material fosilizado, no creemos que esta se encuentre extinta, ya que además de haber sido datada recientemente (i.e. 2865 años de antigüedad), fue colectada en un área muy poco explorada, por lo que probablemente aún habita la Isla Wellington y alrededores.

\section{Nerthra praecipua Todd, 1957}

Esta es probablemente la especie más rara de la familia Gelastocoridae presente en Chile. Junto al holotipo hembra cuya localidad es "Chile", solamente se conoce una hembra adicional reportada por Polhemus \& Cekalovic (1998), de la isla de Chiloé. Polhemus \& Cekalovic (1998) ubican a esta especie junto a las especie australianas del complejo "allaticollis". Sin embargo, Cassis \& Silveira (2001) la remueven de este grupo sin otorgarle una clasificación. Actualmente, con disposición del primer macho de la especie, la ubicamos en un nuevo subgénero, el cual describimos a continuación:

Rhinodermacoris Faúndez subgen. nov. (Figs. 3-8) Especie tipo: Nerthra praceipua Todd, 1957

\section{Diagnosis}

Ocelos ausentes, pronoto rectangular, hemiélitros fusionados, esternito VII entero, genitalia femenina en posición dorsal.

\section{Descripción}

Coloración general: parduzco-anaranjado, con marcas oscuras en los hemiélitros por sobre las venas, superficie en general levemente verrugosa.

Cabeza: Subtriangular en vista frontal, ápice con un pequeño tubérculo, ojos redondeados a ovalados, presencia de tubérculos superapicales y laterales, ocelos ausentes, rostrum corto, no sobrepasando la procoxa.

Tórax: Pronoto fuertemente rectangular (Figs. 3, 4), disco pronotal elevado con forma trapezoide; escutelo subtriangular, con dos carenas laterales gruesas; hemiélitros enteramente coriáceos y fusionados, no sobrepasando el final del abdomen, venas fuertemente marcadas; pleuras con abundantes puntuaciones verruciformes de coloración parda.

Abdomen: Conexiva fuertemente ondulada; segmentos ventrales simétricos; espiráculos con el peritrema marfileño, situados en el borde exterior superior de un parche negruzco. Genitalia femenina (Fig. 5): Esternito VII prolongado posteriormente, no emarginado; segmentos del ovipositor ligeramente asimétricos ubicados posterodorsalmente. Genitalia masculina (Figs. 6, 7, 8): Segmentos pregenitales asimétricos (Fig. 6), segmento VII con un lóbulo lateral bien producido, segmento VIII con una amplia escotadura en V seguida de una división media; pigóforo con forma de cápsula, amplio y con borde redondeado; parámero (Figs. 7, 8) alargado y levemente curvo, con una leve expansión lateral aplanada, que comienza en el primer tercio de la estructura y se extiende hasta el ápice, dándole un aspecto lanceolado.

Dimorfismo sexual: Macho con tubérculos cefálicos menos notorios en la hembra, pronoto con dos zonas convexas en el margen anterior, y hemiélitros más proyectados apicalmente, cubriendo la genitalia.

\section{Etimología}

Rhinoderma-, por el género endémico de Chile y Argentina de anuros Rhinoderma Duméril \& Bibron, $1841 \mathrm{y}$-coris gr., chinche, debido al 

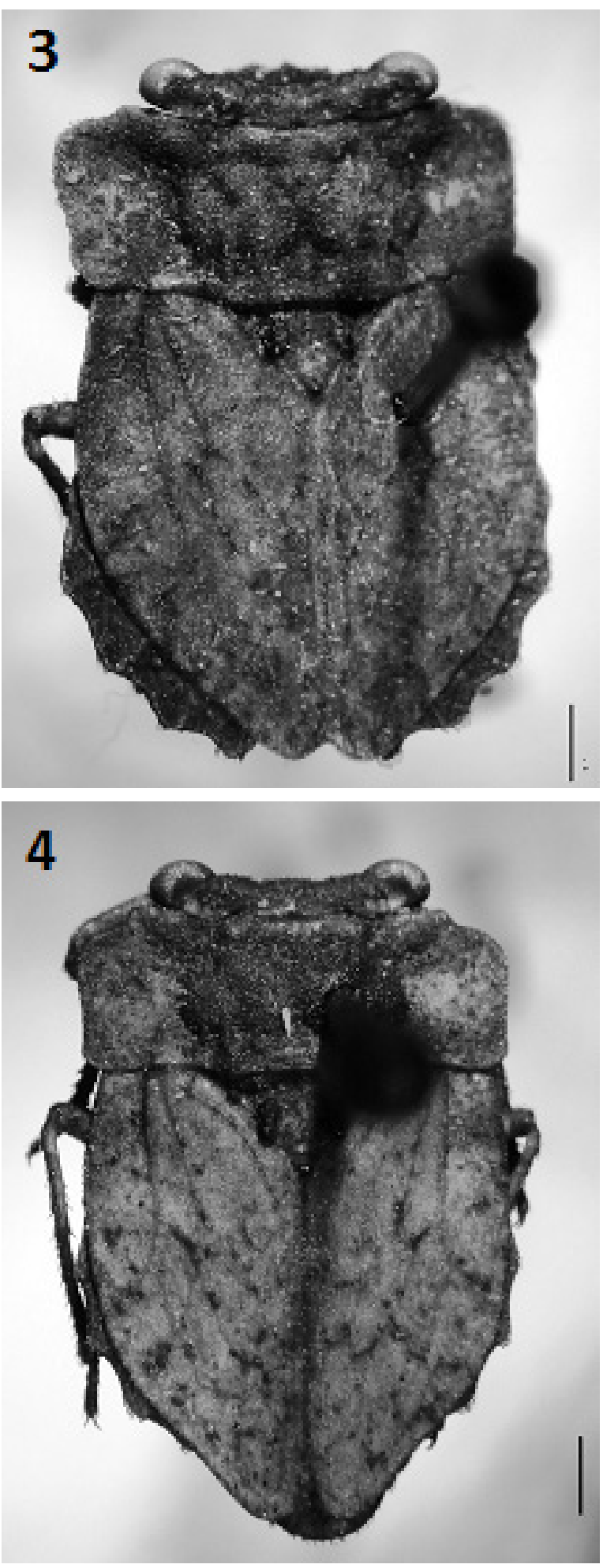

Figuras 3 y 4. Nerthra (Rhinodermacoris) praecipua Todd, 1957 escala $=1 \mathrm{~mm}$; 3. Hembra, habitus; 4. Macho, habitus.
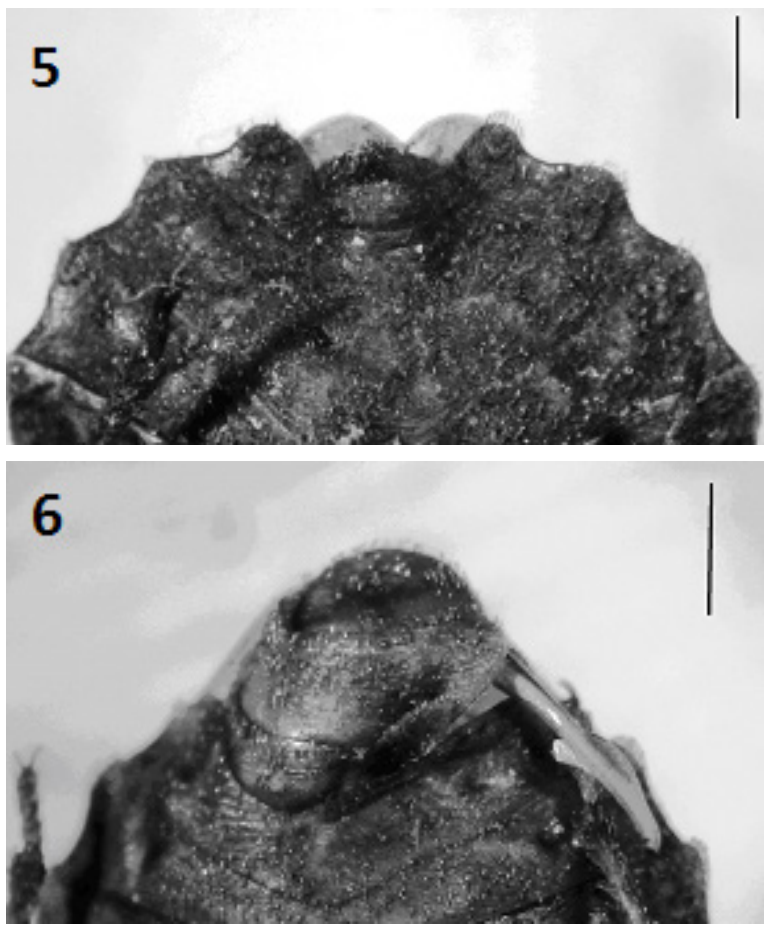

Figuras 5 y 6. Nerthra (Rhinodermacoris) praecipua Todd, 1957 escala $=1 \mathrm{~mm} ; 5$. Terminalia femenina en vista ventral; 6 . Terminalia masculina en vista ventral.

parecido que presenta la especie tipo de este nuevo subgénero con estos anfibios.

\section{Comentario}

Este subgénero se diferencia de Nerthra sensu stricto por la siguiente combinación de caracteres: Ausencia de ocelos, pronoto notablementerectangular, hemiélitrosfusionados, esternito VII prolongado posteriormente y no emarginado (i.e. entero), y la posición dorsal de la genitalia femenina. Como mencionan Polhemus \& Cekalovic (1998), la especie tipo de este subgénero es única en Sudamérica y si bien es cierto que tiene algunas afinidades con especies de Australasia (como del grupo de N. allaticollis y el grupo de $N$. rugosa), ha sido descartado que pertenezca a cualquiera de estos (Cassis \& Silveira, 2001, 2006). Sin embargo, creemos posible que mantengan algún nexo ancestral gondwánico, en los que usualmente las especies sudamericanas suelen ser las más primitivas (e.g. Idiostolidae, Aradidae, Pentatomidae, 

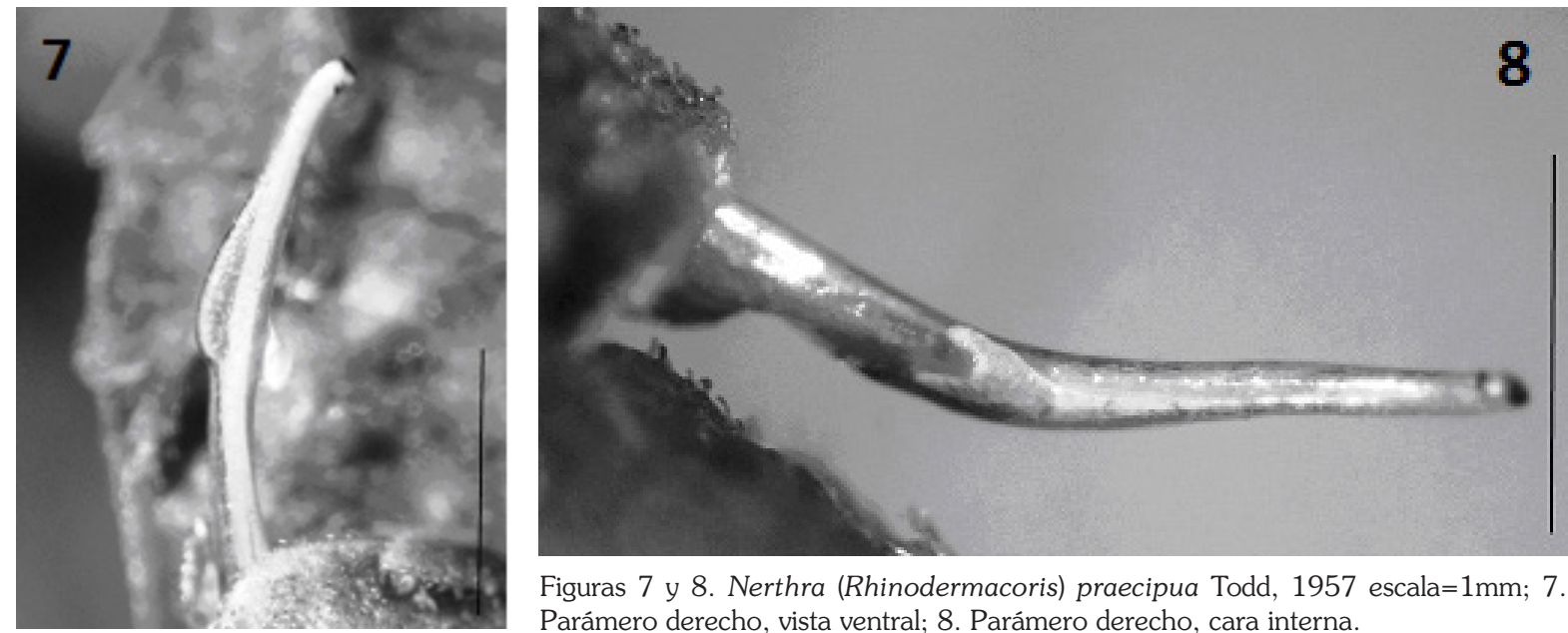

Figuras 7 y 8. Nerthra (Rhinodermacoris) praecipua Todd, 1957 escala=1mm; 7 . Parámero derecho, vista ventral; 8. Parámero derecho, cara interna.

Acanthosomatidae). Finalmente es probable que cuando la familia Gelastocoridae sea revisada a nivel mundial, este subgénero cambie su nivel taxonómico a género, como otros grupos de Nerthra que, de acuerdo a Cassis (2006), deben ubicarse en sus propios géneros. Sin embargo, de momento preferimos mantenerle en un nivel subgenérico.

\section{Material examinado}

Chile, Región de los Ríos, Puente Blanco, $39,542930{ }^{\circ} \mathrm{S} 72,85650{ }^{\circ} \mathrm{O}, 28 / 3-\mathrm{XI}-1987$, open pitfall, A. C. Ashworth leg., 1亏ै [EIFC]; Chile, Región de Los Lagos, Osorno, Pucatrihue San Juan de la Costa, 4/21-II-1967, L. E. Peña leg. 1 우 [MUEC].

\section{CONCLUSIONES}

Los registros aquí entregados representan nuevas localidades para Nerthra (Rhinodermacoris) praecipua, extendiendo su distribución hacia el norte, alcanzando la Región de los Ríos ( 40 S); además la genitalia masculina es descrita por primera vez para esta especie.

La localidad de Puerto Edén $\left(\sim 49^{\circ} \mathrm{S}\right)$ para Nerthra subantarctica sp. nov., es la más meridional para la familia en el continente americano; ya que anteriormente esto correspondía a $N$. (R.) praecipua en Chiloé. Adicionalmente este registro corresponde al primero para la familia Gelastocoridae en la región de Magallanes.

\section{AGRADECIMIENTOS}

Agradecemos a Danilo Cepeda por permitirnos acceso al material depositado en la colección del Museo Entomológico Luis Peña de la Universidad de Chile.

\section{LITERATURA CITADA}

Ashworth, A. C., Markgraf, V., \& Villagrán, C. (1991). Late Quaternary climatic history of the Chilean Channels based on fossil pollen and beetle analyses, with an analysis of the modern vegetation and pollen rain. Journal of Quaternary Science, 6(4), 279-291.

Cassis, G. (2006). A phylogenetic reclassification of the toad bug subfamily Gelastocoridae (Insecta: Heteroptera). In B. Aukema (Ed.), Third Quadrienal Meeting of the International Heteropterist's Society. Abstracts (p. 1). WICC Wageningen, 18-21 July 2006.

Cassis, G., \& Silveira, R. (2006). A new species and first record of a toadbug (Insecta: Heteroptera: Gelastocoridae) from New Caledonia and zoogeography. Russian Entomological Journal, 15, 147-150.

Cassis, G., \& Silveira, R. (2001). A revision and phylogenetic analysis of the Nerthra alaticollis species-group (Heteroptera: 
Gelastocoridae: Nerthrinae). Journal of the New York Entomological Society, 109, 1-46.

Henry, T. J. (2009). Biodiversity of Heteroptera. In R. G. Foottit, \& P. H. Adler (Eds.), Insect Biodiversity: Science and Society (pp. 223-263). Oxford, UK: Wiley-Blackwell.

Skottsberg, C. (1905). Some remarks upon the geographical distribution of vegetation in the colder Southern Hemisphere. Ymer, 4, 40-427

Polhemus, J. T., \& Cekalovic K. T. (1998).
Redescription of Nerthra praecipua (Heteroptera: Gelastocoridae) from Chile. Entomological News, 109(1), 33-36.

Todd, E. L. (1955). A taxonomic revision of the family Gelastocoridae (Hemiptera). University of Kansas Science Bulletin, 37, 277-475.

Todd, E. L. (1957). Five new species of Gelastocoridae with comments on other species (Hemiptera). Proceedings of the Entomological Society of Washington, 59, 145-162. 